\title{
THE INFLUENCE OF PUMPING PARAMETERS IN FLUID-DRIVEN FRACTURES
}

\author{
E. Sarris and P. Papanastasiou \\ Department of Civil and Environmental Engineering \\ University of Cyprus \\ P.O. BOX 20537, 1678 Nicosia, Cyprus \\ E-mail: esarris@ucy.ac.cy, panospap@ucy.ac.cy
}

Keywords: Hydraulic fracturing, Cohesive zone, Poroelastoplasticity, Fracture mechanics.

\begin{abstract}
In the present work we investigate the main pumping parameters that influence a fluid driven fracture in both cohesive poroelastic and poroelastoplastic weak formations. These parameters include the fluid viscosity and the injection rate.

The fracture is driven in weak permeable porous formation by injecting an incompressible viscous fluid at the fracture inlet assuming plane strain conditions. Fluid flow in the fracture is modeled by lubrication theory. Pore fluid movement in the porous formation is based on the Darcy law. The coupling follows the Biot theory while the irreversible rock deformation is modeled with the Mohr-Coulomb yield criterion with associative flow rule. Fracture propagation criterion is based on the cohesive zone approach. Leak-off is also considered. The investigation is performed numerically with the finite element method to obtain the fracture length, opening and propagation pressure vs time.

We demonstrate that the pumping parameters are influencing the fracture geometry in weak formations through the diffusion which creates back stresses and large plastic zones. It is shown that the back stresses act as a barrier and larger net pressures are needed to propagate the fracture. This effect in the poroelastoplastic formation is responsible for the creation of larger plastic zones. These influences for large viscosity or high injection rates result in larger fracture profiles and propagation pressures. These findings may explain partially the discrepancies in net-pressures between field measurements and conventional model predictions.
\end{abstract}




\section{INTRODUCTION}

The hydraulic fracturing process has been employed widely and successfully by the industry to enhance the production of oil and gas from underground reservoirs for many years. Lately the need for new environmental friendly fuels has oriented the research in exploiting unconventional gas reservoirs utilizing novel hydraulic fracturing techniques. Furthermore, based on the applicability of the technique it not surprising that it's one of the most attractive subjects for research by many disciplines. Among the most successful applications of the hydraulic fracturing technique is the preconditioning of ore rock to promote caving, the determination of the insitu stress in rock, the formation of barriers to block contamination, stimulate geothermal reservoirs for heat production and recently the underground storage of carbon dioxide. Hydraulic fracturing is not only a man-made technique, there are also physical processes that present similarities like the magma-driven fractures and the turbulent water flow driving basal fracture in glacier beds $[1,2]$. Recently, some research works have showed that industrial numerical models rely on the ability to simulate fracture propagation under realistic non-uniform insitu conditions so as to predict accurately the complicated fracture geometry with the even more complicated fracture fluid rheology. There is no existing methodology to accurately measure fracture geometry during and after the process thus numerical modelling is of great importance $[3,4]$.

A large number of studies in hydraulic fracturing have been devoted to the theoretical understanding the near tip effects [4-6]. These contributions have helped in the establishment of scaling framework by formulating mathematically the fluid driven problem to obtain analytical and semi analytical solutions. The focus of their work was based on the injection of incompressible Newtonian or non-Newtonian fluids in elastic impermeable and permeable formations for simple fracturing geometries like the plane strain KGD or the PKN and the axisymmetric penny shape models. The asymptotic solutions obtained for these idealized models serve as a benchmark for numerical simulations. These research works have identified two competing energy dissipation mechanisms and two competing storage mechanisms. In the first case, energy is dissipated in creating and extending the fracture in the porous formation and by viscous flow inside the fracture. In the second case, the storage mechanisms are the fluid leak-off stored in the porous formation and the fluid stored in the fracture. Asymptotic solutions of such processes generally correspond to a fracture driven under limiting conditions or when one of the energy dissipation mechanisms associated with the viscous fluid flow, the toughness of the material, the fluid storage in the formation or the fluid storage in the fracture can be neglected when another dominates. These asymptotic regimes are as follows [4]: (i) storage toughness, (ii) storage viscosity, (iii) leak-off toughness (iv) leak-off viscosity. Nonetheless, due to the significant limitations of the analytical models listed above, numerical models are utilized to overcome the rigorous mathematical difficulties that are associated with the existence of the singularity at the fracture tip and the fluid lag region of the fracture by using the cohesive zone approach. The cohesive fracture growth has been extensively used to model fracture growth in porous and non-porous materials.

The usual parameter that is available and can be measured in the field is the wellbore pressure and it is not surprising that many contributors have devoted effort in its accurate prediction during a treatment. Standard fracture simulators usually underestimate this parameter as they do not account for irreversible effects (poroelastoplastic). A research on net pressures (difference between fracturing fluid pressure and the far-field confining stress) indicated that the net pressures encountered in the field can be 50-70\% higher than the predicted ones [7]. This dictates the importance of the poroelastoplastic behavior of weak rocks in hydraulic fracturing. Recently, a research work based on numerical studies has showed that accounting for 
poroelastoplasticity in the simulations, the fracture width is $22.25 \%$ wider than the elastic fracture and $15.2 \%$ wider than the poroelastic. Accordingly, the pressure needed to propagate a poroelastoplastic fracture, at wellbore, is $20.9 \%$ higher from a poroelastic and $31.8 \%$ higher from the elastic fracture [8]. All these studies had ignored the influence of the pumping parameters and its effects on the porous rock deformations.

In this research work we extend the recent work of [8] in order to evaluate the influence of the pumping parameters of the diffusion process in fluid driven fractures for both poroelastic and poroelastoplastic conditions. The yielding mechanism used in the present work is associated with frictional sliding, either along particles or micro-cracks. The rock is modeled by the Mohr-Coulomb flow theory, the fracture propagation criterion used is the cohesive zone law, the fluid flow inside the fracture is described by lubrication theory and the pore fluid movement inside the porous domain is successfully modeled with the Darcy law. With the solution of the numerical model described above we have found that the pumping parameters affect the diffusion process in the cases examined, poroelastic and poroelastoplastic. The diffusion process which is a major mechanism in hydraulic fracture operations influences further the obtained results on the fluid pressures, fracture dimensions and plastic yielding. Furthermore results are also influenced by the transition from the fracture toughness dominated regime to the viscosity dominated regime. This is another important feature of our numerical model for predicting fractures in both fracture toughness and viscosity dominated regimes. The results obtained are important for improving numerical simulators of modeling hydraulic fracturing in particular. We focused on short fractures in weak formations as such fractures can be used for sand control applications. Furthermore, these results can be used in the prediction of vertical fractures and containment in shale-natural gas stimulations where there are serious environmental concerns on the risk of ground-water contamination.

This paper is organized as follows: in the second part, we describe the governing equations that are involved in the physical processes: the fluid flow, the rock deformation, the fracture propagation, the methodology that was adopted in the numerical model and the scaling of the plastic zones. In the third part, we present and critically evaluate the numerical results and highlight the conclusions on the important parameters.

\section{MODELING METHODOLOGY}

In a typical hydraulic fracturing treatment, the fracturing fluid is blended with proppant material and injected into the wellbore that is appropriately cased and perforated so as to fracture the porous reservoir in preferential paths. When the injection of the fracture blend is performed at high rates, the pressure that is build up splits and drives the fracture hydraulically in the direction where the energy needed to create newly surface is minimized and usually this direction is perpendicular to the minimum insitu stress $[1,8]$. The construction of numerical models that will describe accurately the aforementioned process calls for understanding the involved four important coupled processes $[9,3,4]$. These coupled phenomena include: (i) fluid flow inside the fracture pressurizes the fracture faces and the porous formation deforms; (ii) viscous fluid flow inside the fracture; (iii) fracture and porous formation exchange fluids; and (iv) fracture propagation is a moving boundary problem. Therefore, simulating the above processes requires the solution of a system of non-linear partial differential equations that are associated with the above coupled processes.

The objective of this research work is to investigate the main parameters influencing the diffusion process when the fracture dominated regime goes from toughness to viscosity dominance and furthermore how the diffusion process caused from this situation affects the development of the plastic zones. The leak-off is treated as unidirectional (1D-isotropic) and the scope is to investigate any effects associated with the coupled processes such as changes in 
the deformation due to diffusion of fracture and pore fluid and how the flow and pressure is influenced by mechanical deformation. Additionally, the fluid losses that surround the fracture tip area and the bulk of the fracture are capable to generate back stresses. This effect cannot be ignored in hydraulic fracture operations as these non-linear mechanisms are capable to generate excessive pore pressures or alter the fluid characteristics of the fracturing fluids. Furthermore, they may explain the differences observed in net-pressures between field measurements and model predictions.

\subsection{Cohesive zone model}

There are few studies utilizing the cohesive zone model in fluid driven fractures. Some important studies in this area include the important work of [10] in which they used the cohesive zone approach to model the fracture process in impermeable and permeable rocks. Other important contributions are those of Papanastasiou $[11,12]$ in which he has successfully modeled coupled fluid-solid driven fractures and linked the net pressures with plastic yielding associated with the fracture process. Hydraulic conductivity from the fracture towards the formation was analyzed by [13] which they proposed the influence of the transversal conductivity with the aim of applying these elements in hydro-mechanical diffusion problems. Recently, [14,15] have performed numerical simulations based on a variable stiffness bilinear traction separation model to investigate the influence of the cohesive zone in hydraulic fracturing in both, porous and non porous weak formations. In another study, [16] used the cohesive zone model to analyze viscosity dominated plane strain and penny shape hydraulic fractures. An enhanced three nodded cohesive element was developed by [17] to model hydraulic fractures in permeable formations. They further analyzed the influence of permeability, fracture fluid viscosity and leak-off on a poroelastic fracture and validated their model with asymptotic solutions.
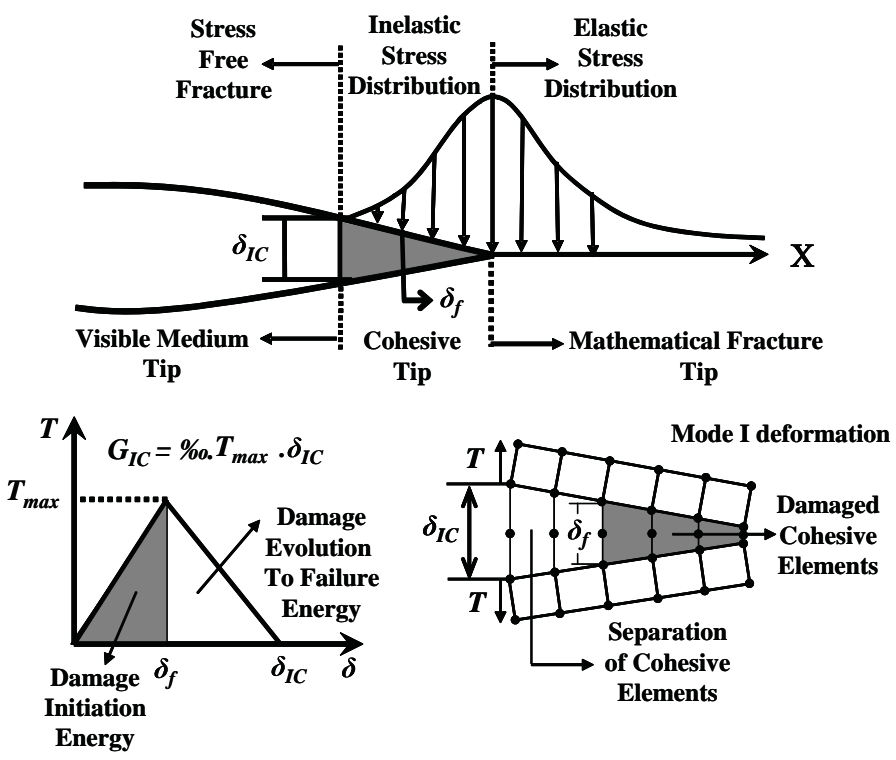

Figure 1: Representation of the cohesive fracture process

As stated in the abundant literature, the concept of the cohesive zone is laid on the fact that the tractions can be transferred through the fictitious fracture faces in the cohesive zone, also called process zone (Figure 1). If analyzed in the micro-mechanical scale, this process zone is the local zone ahead of the fracture tip where micro voids and micro cracks initiate and grown until coalesce with the main body of the fracture. 
The transferred normal traction is a function of the separation and falls to zero at a critical opening and then the fracture propagates. The evolution of the fracture is governed by energy balance between the work of the external loads and the sum of the bulk energy of the unfractured part and the energy dissipated in the fracture process (figure 1). The constitutive behavior of the cohesive zone is defined by the traction-separation relation derived from laboratory tests. The traction-separation constitutive relation for the surface is such that with increasing separation, the traction across this cohesive surface reaches a peak value and then decreases and eventually vanishes, permitting for a complete separation.

Simple cohesive zone models can be described by two independent parameters which are usually, for mode-I plane strain, the normal work of separation or the fracture energy $G_{I C}$ and either the tensile strength $\sigma_{t}$ or the complete separation length $\delta_{I C}$. An additional parameter in these models is the slope of the initial loading which may define a range from rigidsoftening to elastic-softening response under tensile stress-state. The area under the tractionseparation curve equals with fracture energy $G_{I C}$ which is the work needed to create a unit area of fully developed fracture. Rice has showed that the critical value J-integral can be equivalent with the critical value of the fracture energy $J_{I C}=G_{I C}$, when the size of the cohesive zone is small compared to the fracture length. For elastic solids this energy is related to the rock fracture toughness $K_{I C}$ through [18]:

$$
K_{I C}=\sqrt{G_{I C} \cdot E^{*}}
$$

where $E^{*}$ is the plain strain modulus E/(1- $\left.v^{2}\right)$. The rock fracture toughness can be calculated from laboratory tests. For the case of the rigid-softening behavior the traction-separation relation is uniquely determined by:

$$
\sigma=\sigma_{t}\left(1-\delta / \delta_{I C}\right)
$$

where $\sigma_{t}$ is the uniaxial tensile strength of the rock and $\delta_{I C}$ is the critical opening displacement at which $\sigma$ falls to zero. The value of $\delta_{I C}$ is given by:

$$
\delta_{I C}=\frac{2 K_{I C}^{2}\left(1-v^{2}\right)}{\mathrm{E} \sigma_{t}}
$$

For the case of the elastic loading the cohesive constitutive relations were augmented and modified to take into account the initial part of the curve as follows [8]:

$$
\sigma=\sigma_{\mathrm{t}}\left(\delta / \delta_{\mathrm{el}}\right)
$$

With the limit of elastic deformation is given by:

$$
\delta_{\mathrm{el}}=\sigma_{\mathrm{t}} / \mathrm{k}_{\mathrm{n}}
$$

where $k_{n}$ is the stiffness of the traction-separation relation in the loading regime with units of $[\mathrm{MPa} / \mathrm{m}]$. In the post-peak softening regime the cohesive constitutive relation is given by:

$$
\sigma=\sigma_{\mathrm{t}}\left[1-\frac{\left(\delta-\delta_{\mathrm{el}}\right)}{\left(\delta_{\mathrm{IC}}-\delta_{\mathrm{el}}\right)}\right]
$$


The assumption that the cohesive zone localizes, due to its softening behavior, into a narrow band ahead of the visible fracture is very convenient for finite element analysis where the softening behavior can be modeled by cohesive zone elements $[8,14,15]$.

\subsection{Governing equations of fluid flow}

The mechanical response of porous formations is usually complicated by irreversible deformation coupled with diffusion of pore fluid. However, minimizing the effect of the pore fluid diffusion to investigate the influence of the irreversible deformations represents only a small part of the problem. Furthermore, the fluid driven problem in an inelastic saturated porous media further complicates the analysis as the diffusion of the fracturing fluid in the rock formation introduces some rate-dependency in the overall solution and behavior of the numerical model. Thus it is necessary to develop an appropriate numerical model to further investigate the coupling of pore pressure diffusion with non-linear rock deformation and damage criteria.

The type of flow constitutive response comprises as longitudinal and transversal flow along the fracture walls $[13,19]$. The numerical model for the fluid flow is constructed for the complete length of the predetermined fracture path. There is one to one correspondence between the plane strain "flow" and "domain" elements at the corner nodes along the fracture path, ensuring that the fluid mass is conserved across their surface. The lubrication theory assumes laminar flow (uniformly viscous incompressible Newtonian) and it accounts for the time dependent rate of the fracture opening. The continuity equation which imposes the conservation of mass in one dimensional flow in the longitudinal direction is as follows:

$$
\frac{\partial w}{\partial t}+\frac{\partial \mathbf{q}}{\partial x}+q_{i}=Q
$$

where $\mathbf{q}$ is the mass flow rate along fracture length $x, q_{i}$ is the fluid transverse loss in the rock mass, $w$ is the fracture opening and $Q$ is the injection rate (B.C).

For a fluid flow between parallel plates the lubrication equation relates the pressure gradient to the fracture width for a Newtonian fluid of viscosity $\mu$, yields from the conservation of momentum balance:

$$
\mathbf{q}=v \cdot w=-\frac{w^{3}}{12 \mu} \frac{\partial p}{\partial x}
$$

where $p$ denotes the fluid pressure and $v$ the average velocity of the fluid on a cross-section in the fracture. At this point the effective channel viscosity $\mu^{*}$ can be defined related to the fluid viscosity through the relation $\mu^{*}=12 \mu$. Equation (8) determines the pressure profile along the fracture from the local width and local flow rate.

The transverse flow which describes the leak-off from the fracture to the surrounding formation is evaluated as a localized potential drop by using a six node cohesive element (figure 1). In these elements, the boundary nodes are shared with the nodes of the adjacent continuum formation so that the nodes of the continuum elements represent the potentials in the pore system on each side of the cohesive interface. The central nodes of the cohesive elements represent the average potential of the fluid in the fracture considered as a channel. According to this distribution of hydraulic head within the cohesive elements, two different potential drops exist as [13]:

$$
q_{t}^{u}=k_{t}^{u}\left(h^{u}-h^{i}\right) \quad q_{t}^{d}=k_{t}^{d}\left(h^{d}-h^{i}\right)
$$


where $q_{i}$ is the transversal fluid loss, $k_{t}$ is the transversal transmissivity, $h^{i}$ is the hydraulic head inside the fracture. The superscript $u$ and $d$ stand for up and down element faces respectively. Combining equations (7-9) we obtain the Reynolds lubrication equation as [20]:

$$
\frac{\partial w}{\partial t}+k_{t}^{u}\left(h^{u}-h^{i}\right)+k_{t}^{d}\left(h^{d}-h^{i}\right)=\frac{1}{12 \mu}\left(\frac{w^{3} \partial p}{\partial x}\right)+Q
$$

The terms $h^{i}, h^{u}$ and $h^{d}$ are uniquely related to the fluid pressure gradients that are actually the tractions acting on the open surfaces of the cohesive elements. According to equation (10) as the cohesive elements undergo complete failure, the cohesive tractions vanish and there is no contribution from the cohesive elements on the open part of the fracture. The fluid pressure which opens the fracture is balanced by the far field stress acting across the cohesive zone and by the cohesive tractions still acting on that zone thus effectively avoiding the singularity at the fracture tip. The only constrain in equation (10) is that the tractions acting on the entire fracture and cohesive zone must be in equilibrium [16].

For the numerical solution we define a nominal small initial width and a small initial length as initial conditions. Generally, the pressure along the fracture in not known a priori and it is a part of the solution. The position of the fluid-front is defined by the mass conservation in the fracture. The behavior of the fluid movement thought the saturated porous formation with respect to the solid, is assumed that obeys the Darcy law. This classical transport law for isotropic porous media relates $q$ to the gradient of the fluid pressure $p$ according to:

$$
q=-(k / \mu)(\nabla p-f)
$$

where $q$ is the fluid flux, $k$ is the intrinsic permeability (assumed to be constant), $\nabla$ is the gradient operator and $f$ are the fluid volume forces. Note that we assume that the fluid in the fracture is identical rheologically to the pore fluid.

\subsection{Rock deformation}

The inelastic rock behavior is a function of the material parameters and loading conditions. Examining the near tip area of the fracture, due to the high stress concentration, solutions from LEFM cannot accurately analyze the fracture process. In conventional rock mechanics, failure of rock formations is analyzed for dry domains (i.e. no pore pressure acting in the system). However, the usual assumption when analyzing failure of rocks in poromechanical systems is that the rock is fluid saturated. Since the formations in petroleum related rock mechanics are generally saturated, this assumption is quite valid and it is important to investigate how saturation influences the failure process. The total stresses $\sigma_{i j}$ are related to the effective stresses $\sigma_{i j}^{\prime}$ through:

$$
\sigma_{i j}=\sigma_{i j}^{\prime}-a p
$$

where $p$ is the fluid pressure, $\alpha$ is the poroelastic constant $a=1-\left(K_{f} / K_{s}\right), K_{f}$ is the fluid bulk modulus the $K_{s}$ is the solid grain bulk modulus. The effective stresses govern the deformation and failure of the rock. In this case the failure criterion is modified to account the fluid pressure constrain by introducing the effective stress into the dry form of the failure criterion. The modified Mohr-Coulomb criterion describing failure in porous conditions is [15]:

$$
\sigma_{1}-p_{f}=2 c \frac{\cos \varphi}{1-\sin \varphi}+\left(\sigma_{3}-p_{f}\right) \frac{1+\sin \varphi}{1-\sin \varphi}
$$


where $\sigma_{l}$ is the maximum insitu stress, $\sigma_{3}$ is the maximum insitu stress, $p_{f}$ is the pore pressure acting in the system, $c$ is the material cohesion and $\varphi$ is the material friction angle. The sign convention adopted here is according to classical mechanics i.e compression is negative.

The only extra parameters that are needed to model the inelastic behavior of the porous continuum as described above are the material cohesion $c$, the material friction angle $\varphi$ and the dilation angle $\psi$, that controls the volumetric change due to yielding and dilation of the material. However, in order to keep the parameters to a minimum, we have assumed associative plasticity theory which justifies the equivalence of the material friction angle with the dilation angle. Furthermore, in the hydraulic fracturing problem, the initial in-situ mean pressure in the near area of the fracture tip decreases during propagation and under such conditions it is reasonable to assume an associative behaviour. Summarizing, the rock mass remote from the fracture is initially elastic, then deforms plastically and then unloads elastically after the fracture has advanced.

\subsection{Propagation regimes and plastic zone scaling}

The fluid driven problem with leak-off in a poroelastoplastic formation is characterized by the four controlling parameters. (i) the volumetric injection rate $Q$, (ii) the fluid viscosity $\mu$, (iii) the leak off coefficient $C$ and (iv) the plane strain modulus $E$. Furthermore, the leak off coefficient is influenced by the minimum insitu stress $\sigma_{0}$ through [17]:

$$
C_{L} \approx \frac{k}{\mu} \frac{\sigma_{0}}{\sqrt{\pi c}}
$$

Where $k$ is the intrinsic permeability, $\mu$ is the fluid viscosity, $\sigma_{0}$ is the minimum insitu stress and $c$ is the poroelastic intrinsic diffusion coefficient which is a function of the Biot modulus $M$, shear modulus $G$, drained $K$ and undrained $K_{u}$ bulk modulus. Equation (19) implies that large insitu stress and permeabilities will result in high fluid losses in the formation. In a similar way small values of fluid viscosity will also result in high fluid losses. According to [6] the fracture propagation is governed by two energy dissipation mechanisms (rock splitting and viscous fluid flow) and two storage mechanisms (inside the fracture and in the porous formation). The relative magnitude of the dissipation processes is characterized by the dimensionless toughness $K_{d}$ and reflects the ratio of energy rate expended in fracturing the rock to viscous dissipation. The dimensionless toughness is linked to the dimensionless viscosity $M_{d}$ and reflects the energy expended in the viscous fluid flow $[6,4]$.

$$
K_{d}=\frac{8}{\sqrt{2 \pi}} k_{i c}\left(\frac{1}{12 \mu Q_{o}\left(E / 1-v^{2}\right)^{3}}\right)^{1 / 4}, \quad M_{d}=\left(K_{d}\right)^{-4}
$$

From the above relations we can determine whether the fracture is driven in the toughness $K_{d}>1$ or viscosity $M_{d}>1$ dominated regime.

The characteristic length of a fracture driven in an elastoplastic formation that relates the material properties and the fluid rheology parameters with the size of the plastic zones is given by [21]:

$$
\ell_{p} \sim \frac{E^{\prime 2}(\mu v)}{\sigma_{T}^{3}}
$$

where $E$ ' is the plane strain modulus, $\mu$ is the fluid viscosity, $v$ is the propagation velocity and $\sigma_{T}$ is the tensile yield strength. According to this characteristic length, high values of elastic 
modulus, viscosity, propagation velocity and low values of rock strength will lead to higher stress concentrations near the tip and higher plastic zones are expected to develop. The dominant term in the viscous fluid flow is $(\mu v)$ and is the aim of our investigation with regarding the influence of the pumping parameters on the resulting plastic zone development.

\section{NUMERICAL ANALYSIS}

The system of equations describing the fluid driven problem was solved with the finite element method. A sufficient fine mesh around the predefined fracture path was used to ensure numerical accuracy from the sharp changes that are created in the geometry of the propagating tip. The theoretical bounds for the cohesive element size was originally discussed by [18] and improved by [22]. According to their work, two main factors influence the element size. First is the cohesive zone size $d_{z}$ and has to be small enough to accurately resolve the stress distributions in the cohesive process zone around the fracture tip. The cohesive zone is an inherent length scale determined by material parameters and it is a very small length scale in most cases. The second factor is the macroscopic stiffness reduction due to the cohesive separation along element boundaries if the initial stiffness of the cohesive surfaces is finite. The cohesive element size must be chosen to ensure element size independency from the aforementioned factors in fracture propagation simulations. The allowable range of element size $d_{z}$ in plane strain conditions is given by:

$$
d_{z}<<\frac{9 \pi G_{I C} E^{\prime}}{32 T_{\max }^{2}}
$$

where $G_{I C}$ is the fracture energy for mode-I separation, $E$ ' is the plane strain modulus, $T_{\max }$ is the maximum traction.

Calculations were carried out in Abaqus (2010), a nonlinear finite element code suit of programs. The usual 4-node, plane strain, isoparametric elements were used to model the domain and 6-node cohesive elements to model the fluid flow in the fracture and the fracturing process. Both types of elements, additionally from their $\mathrm{u}, \mathrm{v}$ translation degree of freedom (D.O.F), are equipped with a pore pressure D.O.F to account for the fluid diffusion in the porous domain. The two additional nodes in the cohesive elements, positioned in their center, are used to simulate the longitudinal and transversal fluid flow during propagation. The descritized domain was considered to be $30 \times 30 \mathrm{~m}$ and the predefined path of the fracture was defined to be $11 \mathrm{~m}$ only (cohesive elements) in order to save computational time. The wellbore location is at the left-lower corner and the fracture is assumed to grow in both directions along the axis-x. For a long fracture the size of the wellbore is negligible and is usually ignored in the modeling. This remark, along with the condition that the wellbore is cased, cemented and fully bonded with the rock formation, justifies the use of symmetry conditions within a reasonable accuracy. Symmetry conditions were imposed at the bottom $(\mathrm{dy}=0$, fixed displacements) and at the left ( $\mathrm{dx}=0$, fixed displacements) side of the models. The in-situ stresses were generated as initial stresses and by applying the equilibrium load at the far end edges. At the top edge the value of $\sigma_{3}(\mathrm{MPa})$ was considered as the minimum in situ stress and at the right side of the models the value of $\sigma_{1}(\mathrm{MPa})$ was considered as the maximum in situ stress. An initial condition is also required for defining an initial fracture length for the flow. This length was considered $0.1 \mathrm{~m}$ which is approximately equal with the perforation length. 


\subsection{Model validation}

The first part of this investigation is devoted to the presentation of the results from the analysis of the fluid driven problem propagating in a poroelastic formation with fluid losses to demonstrate the fully coupled solution. We have performed comparisons with the asymptotic solution of [9] and the numerical computations of [17] when the fracture is driven in a fracture toughness dominant regime. We have also compared with the asymptotic solution of [6] and the numerical computations of [17] when the fracture is driven in a viscosity dominant regime. The parameters of the numerical computations to be consistent with the numerical results of [17] are as follows: Young modulus, $17 \mathrm{GPa}$; Poisson ratio, 0.2; Fracture energy for mode-I separation, 120 Pa.m; Critical separation traction, $1.25 \mathrm{MPa}$; Injection rate, 0.001 $\mathrm{m}^{2} / \mathrm{sec}$; Permeability, $10^{-15} \mathrm{~m}^{2}$; fluid viscosity, $0.0001 \mathrm{~Pa}$.sec; compressive far field stress, 3.7 $\mathrm{MPa}$; Porosity, 0.2; Leak-off coefficient, $6.28 \times 10^{-5} \mathrm{~m} \cdot \mathrm{sec}^{-1 / 2}$. The difference between our numerical model and the numerical model of [17] is that we introduce an initial stiffness to the cohesive surfaces that is characterized by an independent parameter. Therefore, the cohesive zone model adopted by [17] here can be regarded as a special case with initially rigid-linear softening bilinear law.
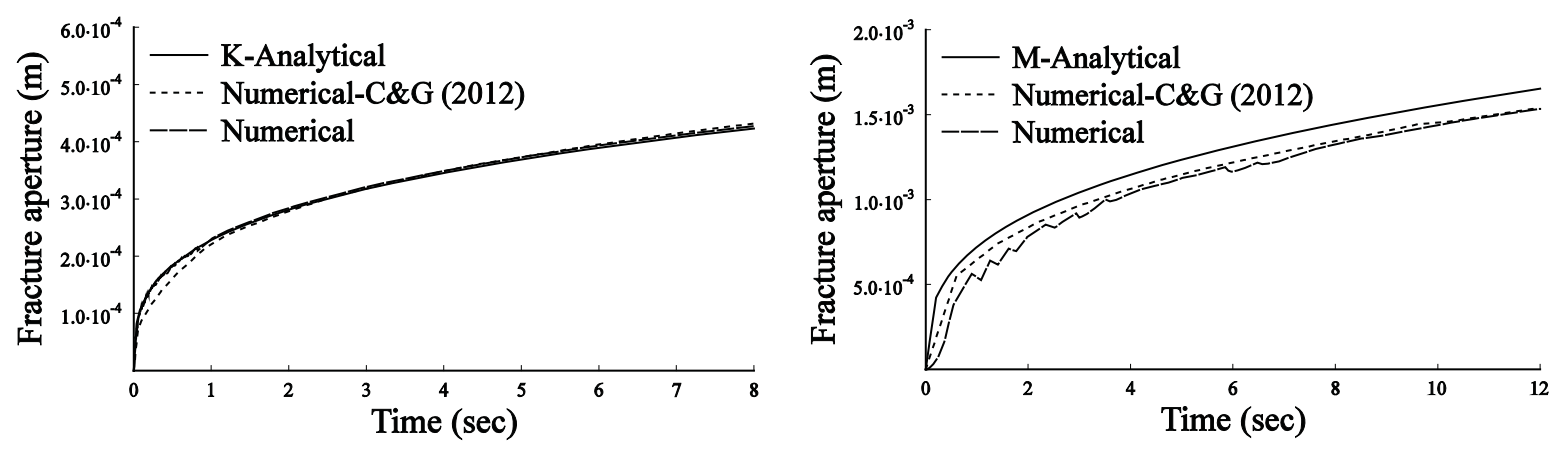

Figure 2: Validation of toughness K-dominated and viscosity M-dominated fractures

Figure (2a) presents the numerical solution of the fracture aperture at wellbore versus time in comparison with the near-K analytical solution of [9] and the numerical solution of [17]. The fracture was left to propagate for 8 seconds (approximately $5 \mathrm{~m}$ long). From the comparison that is performed, it is seen that the agreement between the near-K analytical and the numerical results is very good. Figure (2b) shows the numerical solution of the fracture aperture at wellbore versus time in comparison with the analytical solution of [6] and the numerical solution of [17]. The fracture was left to propagate for 12 seconds (approximately $5 \mathrm{~m}$ long). The fluid viscosity was raised to $0.1 \mathrm{~Pa}$.sec. The results from this simulation are in good agreement between the numerical results of [17] but not so good with the M-analytical solution. The reason for this disagreement is that the analytical solution is based on the assumption of LEFM fracture propagation and leak-off is governed by Carter's law. In the numerical solution, fracture propagation based on the cohesive zone assumption and fluid losses are based on the diffusion equation (equation 10) which is more accurate to the author's opinion. The losses demonstrate the action of back-stresses which induce a negative stress on the fracture walls causing the fracture to close. This effect is in agreement with the numerical numerical solution presented by [17]. A small discrepancy is also observed for early times $(0-3 \mathrm{sec})$ within acceptable limits due to the fracture loading (pumping schedule considered as boundary condition), and the high value of fluid viscosity. The apparent discontinuities that are ob- 
served on the numerical solution are attributed to the mesh resolution. However, no refinement it needed as seen from the convergence with the other solutions presented.

From the above analysis we have showed that our model is capable of simulating fluid driven fractures in both, the fracture toughness and viscosity dominated regimes under poroelastic conditions. We will extend the applicability of the numerical model to account for the poroplastic zone development according to the scaling presented in equation (21).

\subsection{Computational results}

This section presents the computations that were performed to analyze the fluid driven fracture propagating in poroelastic and poroelastoplastic weak formations. The purpose of this investigation is the link of the pumping parameters (fluid viscosity and injection rate) with the plastic zone development while the fracture is driven in both K-dominated and M-dominated regimes. The input parameters which the numerical computations were performed are given in Table 1. These parameters include the rock properties, the pumping parameters, and cohesive zone properties. For the poroelastic case it is assumed that any compressibility effects will not affect the fracture geometry or the fluid pressure (i.e incompressible formations). In both cases we consider an initial pore pressure exist in the formation and is assumed to have a uniform value of $1.85 \mathrm{MPa}$. For the poroelastoplastic case, the only extra parameters that are needed to consider porous deformation and propagation the material cohesion $c$, and friction angle $\varphi$. The cohesive zone is assumed to have a unit normal and anti-plane thickness. The effective insitu stress field was considered as $\sigma_{1}, 14 \mathrm{MPa} ; \sigma_{2}, 9 \mathrm{MPa} ; \sigma_{3}, 3.7 \mathrm{MPa}$. As initial conditions, we have used a void ratio equal to 0.333 and an initial gap (perforation length) equal to $0.1 \mathrm{~m}$.

\begin{tabular}{lr}
\hline Variable & Value \\
\hline Elastic Rock Properties & 16200 \\
\hline Young modulus, $E(\mathrm{MPa})$ & 0.3 \\
Poisson ratio, $v$ & $2.421 \mathrm{E}-10$ \\
\hline Domain hydraulic conductivity, $k(\mathrm{~m} / \mathrm{sec})$ & 1.515 \\
\hline Inelastic Rock Properties & 28 \\
\hline Cohesion, $c(\mathrm{MPa})$ & \\
Friction and dilation angle, $\varphi^{0}=\psi^{0}$ & 0.5 \\
\hline Cohesive zone properties & $162 \mathrm{E}+3$ \\
\hline Maximum Traction, $\sigma_{t}(\mathrm{MPa})$ & 0.112 \\
Cohesive stiffness, $K_{n}(\mathrm{MPa})$ & $2.421 \mathrm{E}-10$ \\
Cohesive energy, $J_{I C}=G_{I C}(\mathrm{kPa} . \mathrm{m})$ & \\
Transverse conductivity $k_{t}^{u}, k_{t}^{d}(\mathrm{~m} / \mathrm{sec})$ & $1 / 5 / 10 / 50 / 100$ \\
\hline Pumping Parameters & \\
\hline Viscosity, $\mu($ Poise $)$ & $0.5 / 5.0 / 10.0 / 15.0 / 20.0$ \\
Injection rate, $Q\left(\mathrm{E}-03 \mathrm{~m}^{2} / \mathrm{sec}\right)$ & \\
\hline
\end{tabular}

Table 1: Input parameters and material properties

The fluid pressure responsible for splitting the formation and driving hydraulically the fracture are generated from the injection rate and is expressed as the fluid loading inside the fracture. For convergence reasons, the injection rate was set to climb its final flow rate value at the first 0.05 seconds of the injection time. This introduces an initial slope in the pumping schedule used; however its effect is considered negligible. The computational results presented next correspond are divided into two categories: influence of injection flow rate and influence of fluid viscosity under poroelastic and poroelastoplastic conditions. 


\subsection{Influence of the injection flow rate}

As explained before, when driving hydraulically a fracture in a porous formation there are four asymptotic regimes. In order to suppress the significant influence of the leak-off toughnees and leak-off viscosity and to study the fracturing process in the toughness and viscosity dominated regimes, we have performed all simulations with a very small value of transversal conductivity and for early times to ensure that the scaling will remain in the near storage regimes (equation 20). At the first part of this investigation, we have kept a constant value of viscosity ( 1 Poise) and perform simulations for different injection rates ranging from $0.5 \mathrm{E}-03$ to $20.0 \mathrm{E}-03$ that correspond to different propagation velocities ranging from slow to fast fracture propagation respectively. According to the scaling of equations (20), this ensures that the fracture will propagate under the toughness dominated regime. This investigation raises the possibility to investigate the parameters affecting the diffusion process in hydraulic operations. All comparisons are made after the fractures where left to reach $1.5 \mathrm{~m}$ long.
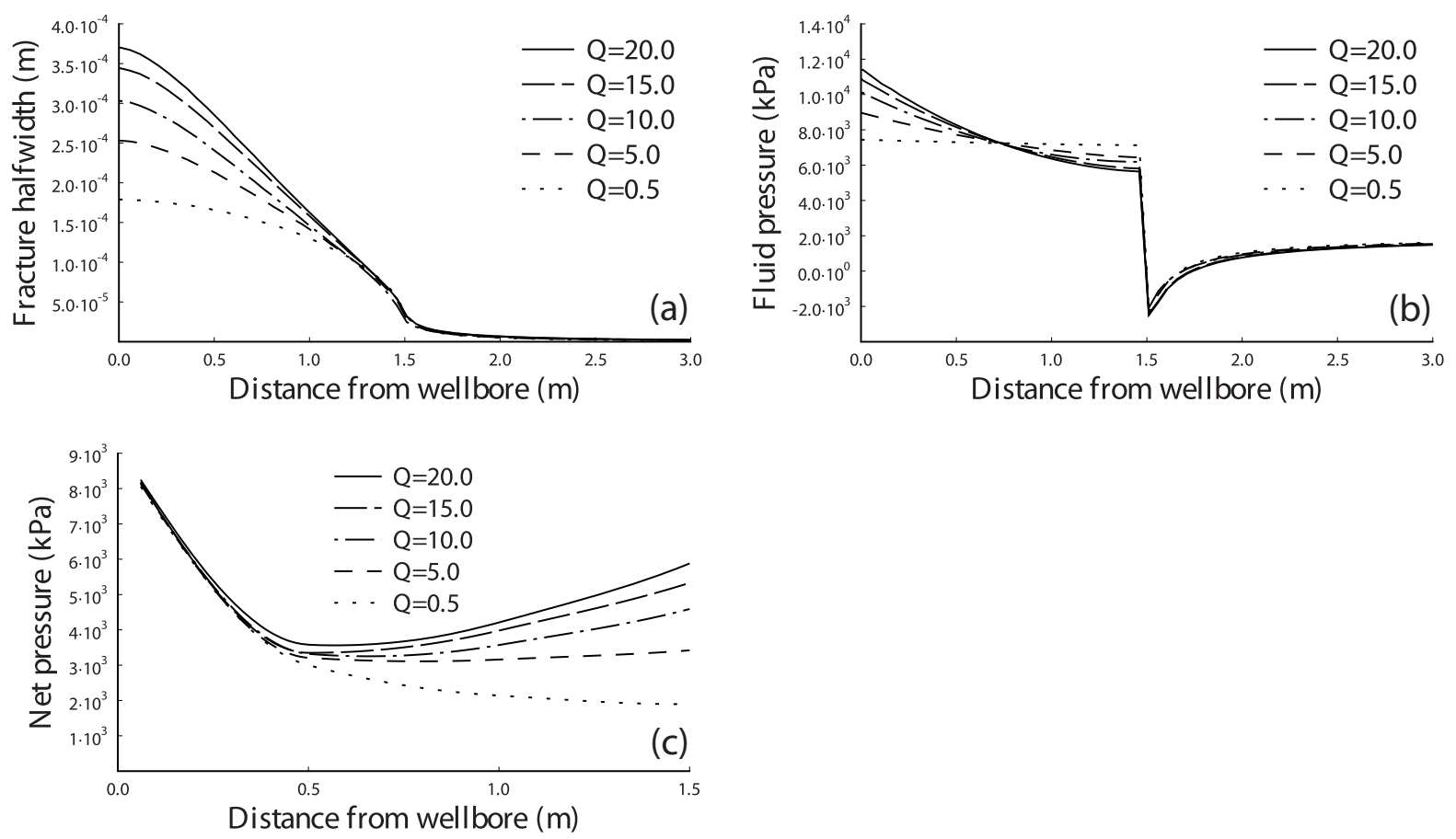

Figure 3: Influence of flow rate under poroelastic conditions

Figure (3a-c) presents the parametric analysis performed for the investigation of the influence of the flow rate under poroelastic conditions. Figure (3a) presents the fracture profiles for the different values of injection rates. It is seen that the fracture is significantly wider when is driven with fast propagation velocity while the aperture is significantly narrow (3 times) when the propagation velocity is slow. This is explained from the small fluid leak-off that is permitted in the models. For the fast propagation case (figure $3 b$ ) it is seen that the fluid pressures turn highly non-uniform and rise inside the fracture as a result of the small diffusion predicted by the model. Accordingly, large loading is observed at the fracture inlet and smaller loading at the fracture tip. This is responsible for the wider profiles. For the slow propagation case, the fluid pressure inside the fracture is practically uniform allowing for larger fluid leak-off and the created back stresses become significant thus producing narrower fracture profiles. Figure (3c) presents the fluid net-pressures. It is showed that the net pressure with the slow propagation velocity after the formation breakdown decreases to a constant val- 
ue as expected. As the fracture is driven with higher flow rates, leak-off is minimized causing the net-pressure to increase. We conclude that the obtained results are a direct result of the time scale difference between the diffusion process and the pumping schedule imposed with the different flow rates.
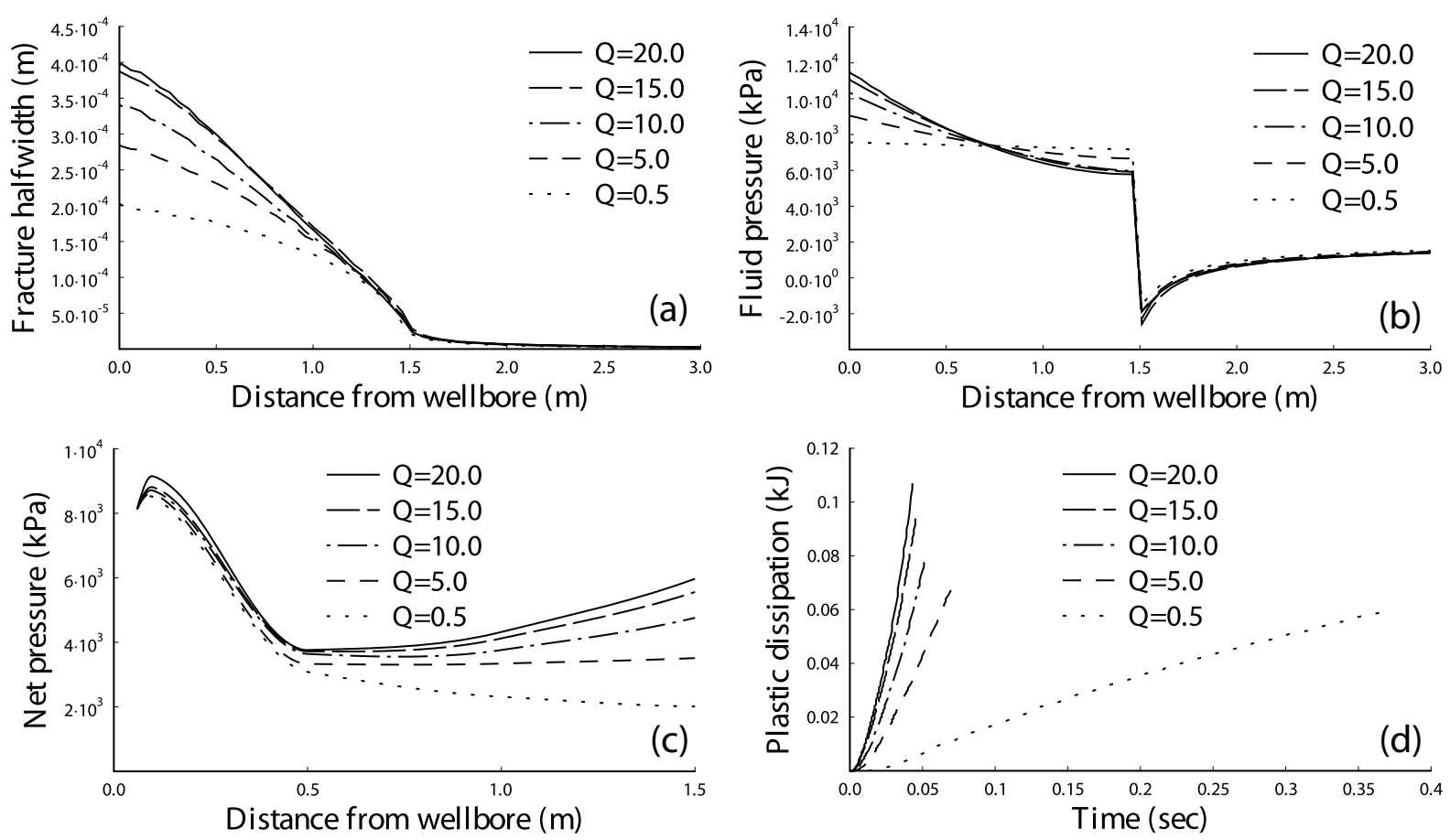

Figure 4: Influence of flow rate under poroelastoplastic conditions

Figure (4a-d) presents the parametric analysis performed for the investigation of the influence of the flow rate under poroelastoplastic conditions. Figure (4a) presents the fracture profiles for the different values of injection rates. It is seen that the fracture is significantly wider when the injection rate is large corresponding to fast propagation velocity. The fracture aperture is significantly narrow when the propagation velocity is slow. This behaviour, as in the poroelastic case, is explained from the small fluid leak-off that is permitted in the models. When the poroelastoplastic fracture is compared with the poroelastic for each injection flow rate it is seen the poroelastoplastic fracture is always wider. This is explained by the action of plastic yielding that the fracture is experiencing. Figure $(4 \mathrm{~b})$ presents the fluid pressure inside the propagating fracture. For the fast propagation case it is seen that the fluid pressure as showed in the poroelastic fracture is highly non-uniform. The value is significantly higher and is observed at the fracture inlet while is smaller at the fracture tip. This action explains the wider profiles. For the slow propagation case, the fluid pressure inside the fracture is practically uniform allowing for larger fluid leak-off and the created back stresses become significant thus producing narrower fracture profiles. When the fluid pressures are compared between the poroelastoplastic and the poroelastic formations, it is seen that in the poroelastoplastic case are always larger as expected due to the influence of plasticity. Figure (4c) presents the fluid net-pressures for the fracture profiles of figure (4a). It is seen that just after the injection starts, pressure starts to build up. This is pressure built up is associated with the plastic zone development. After the plastic zones have fully developed, the pressure starts to decay. This behavior is similar for all cases of injection rates examined. The predicted net pressure with the slow propagation velocity after the formation breakdown decreases to a constant value as expected. As the fracture is driven with higher flow rates, leak-off is minimized 
causing the net-pressure to increase. This is once again a result of the limited diffusion causing the fluid pressure to become non-uniform resulting in wider fracture profiles. As explained earlier, this is a result of the time scale difference between the diffusion process and the pumping schedule. From the elevated net pressures predicted from the numerical model the energy that is dissipated due to plastic yielding versus time for all the cases examined is showed in figure (4d). It is showed that with high injection rates significantly larger plastic dissipation is created in the numerical models. For small injection rates, more time is needed to reach the desired length $(1.5 \mathrm{~m})$ however smaller plastic yielding is created. This result suggest that it is better to propagate a fluid driven fracture with small injection rate in expense of the propagation time because the created hydraulic pressure is uniform and any unnecessary plastic yielding associated with high injection rate to create non-uniform loading inside the fracture is avoided.

\subsection{Influence of the fracture fluid viscosity}

The second part of this investigation is concerned with the influence of the fluid viscosity in the diffusion process and how the plastic zone development is affected when the fracture is driven in the viscosity dominated regime (equation 20). In order to analyze the results we have kept a constant value of injection rate $(0.5 \mathrm{E}-03)$ and perform simulations for different values of fluid viscosity ranging from 1 to 100 Poise. These values of fluid viscosity correspond from small to highly viscous fluid flow. Such values of fluid viscosity (100 Poise) actually confirm a fracture driven in the viscosity dominated regime. We have used the value of the smallest injection rate to minimize any interaction effects between the fluid viscosity and the injection rate scaling term $(\mu . v)$ for the investigation of the plastic zone length as showed in the scaling of equation (21). As in the previous investigation (section 3.3) all comparisons are made after the fractures where left to reach $1.5 \mathrm{~m}$ long.
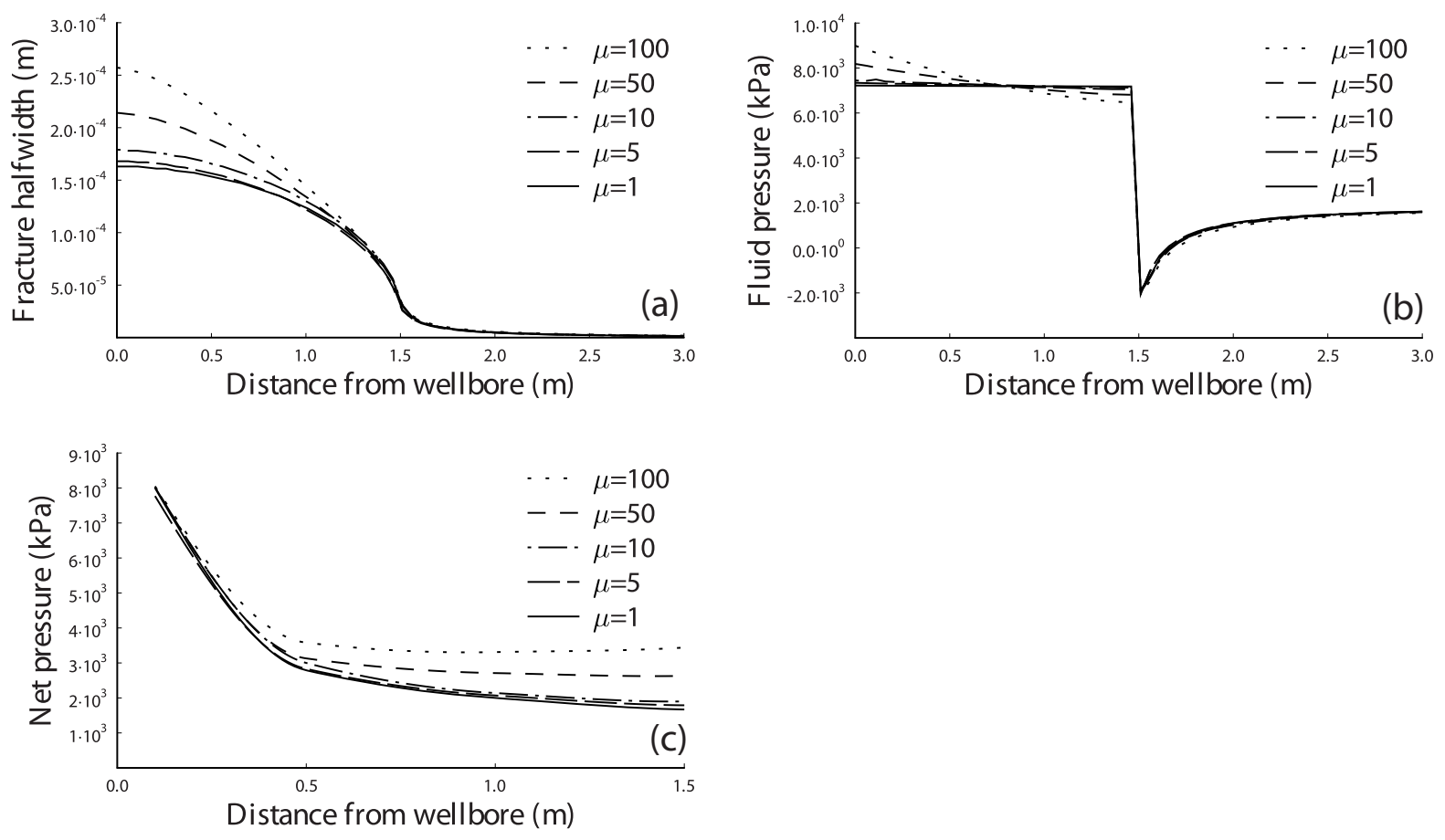

Figure 5: Influence of viscosity under poroelastic conditions 
Figure (5a-c) presents the numerical solution for a viscosity dominated fracture which is driven under poroelastic conditions. The investigated variable is the fluid viscosity. Figure (5a) shows the fracture profiles for the different values of fluid viscosities. From figure (5a) it is seen that for small values of fluid viscosity (1-10 Poise) the action of the viscous fluid flow does not present any significant effects on the fracture profiles. In fact the fractures present similar profile. However, the fracture is significantly wider when is driven with highly viscous fluid while the aperture is narrower ( 2 times) when fracturing is performed with a low viscosity fluid. This is explained from the small fluid leak-off that is permitted in the models through the transverse hydraulic conductivity (filter cake) and the action of the viscous fluid flow. For the case where the fracture is driven with a highly viscous fluid (figure $5 \mathrm{~b}$ ) it is seen that the fluid pressures turn highly non-uniform with the larger loading values observed at the fracture inlet and smaller loading at the fracture tip. This is responsible for the wider profiles. For the other case, where the fracture is driven with a less viscous fluid, the fluid pressure inside the fracture is practically uniform therefore allowing for larger fluid leak-off and as a result the created back stresses become significant resulting in narrow fracture profiles. Figure (5c) presents the fluid net-pressures obtained for the fracture profiles of figure (5a). From this figure $(5 \mathrm{c})$ it is showed that the net pressure predicted with the less viscous fluids after the formation breakdown decreases to a constant value as expected. When the fracture is driven with highly viscous fluid, leak-off is minimized causing the net-pressure to increase. We conclude that the obtained results are a direct result of the action of the viscous fluid flow. This means that higher net and fluid pressures are created and the resulting fracture profiles are wider when the fracture is driven in the viscosity dominated regime. Furthermore, this phenomenon is amplified with the time scale difference between the diffusion process and the pumping schedule imposed with the different flow rates.
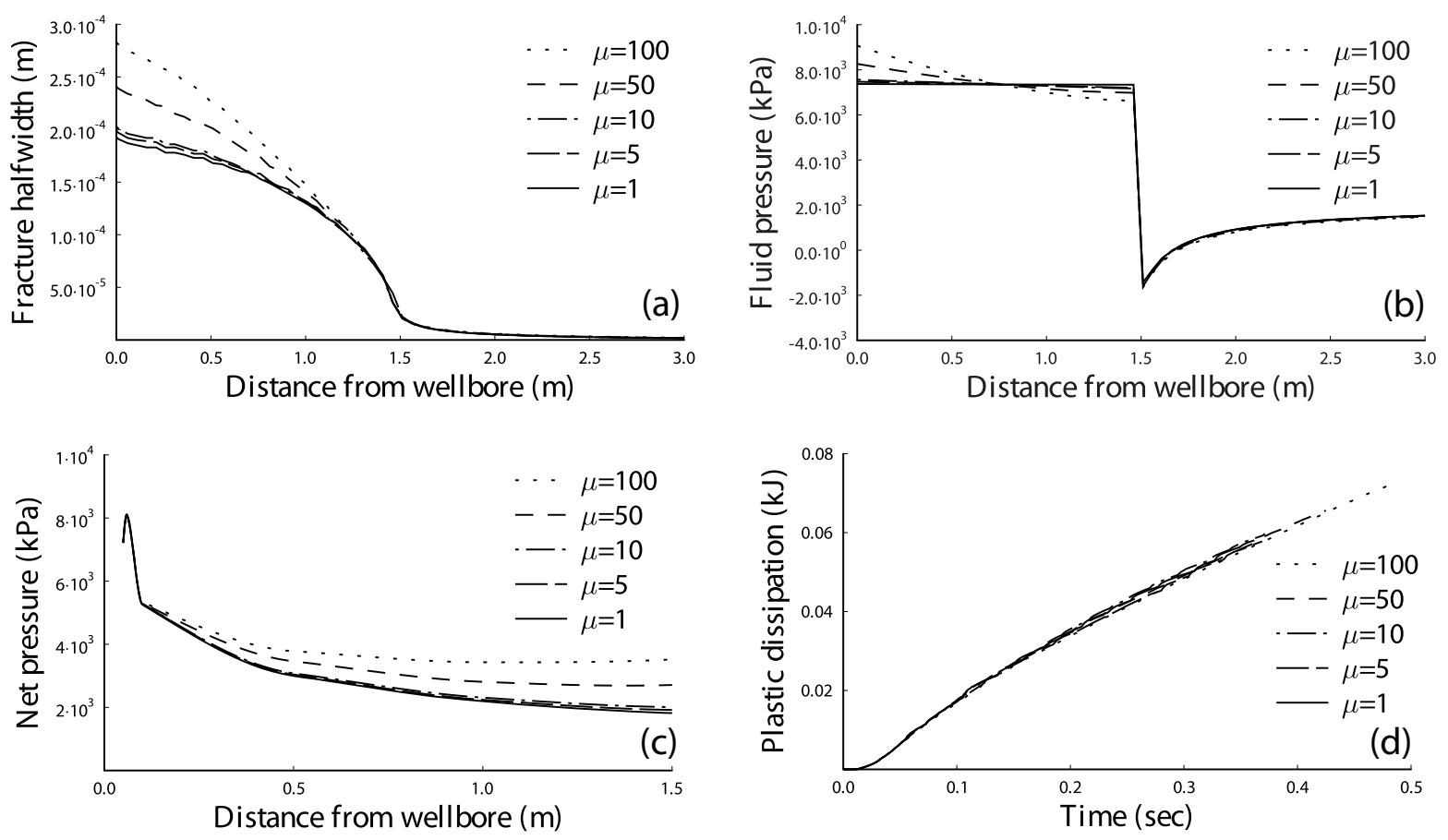

Figure 6: Influence of viscosity under poroelastoplastic conditions

Figure (6a-d) presents the computational results for a viscosity dominated fracture which is driven under poroelastoplastic conditions. Figure (6a) shows the fracture profiles for the different values of fluid viscosities. From figure (6a) it is seen that for small values of fluid vis- 
cosity (1-10 Poise) the action of the viscous fluid flow does not present any significant effect on the fracture profiles. These observations are the same as the poroelastic case. This can be attributed to the set of parameters chosen for these simulations. The fracture is significantly wider when is driven with highly viscous fluid while the aperture is narrower when fracturing is performed with a low viscosity fluid. This behaviour is explained once again, due to the small fluid leak-off that is permitted in the models through the transverse hydraulic conductivity (filter cake) and the action of the viscous fluid flow. When the fractures are compared for all the viscosity cases between the poroelastic and poroelastoplastic fractures, the fractures are always significantly wider in the poroelastoplastic cases. This demonstrates that when irreversible effects are considered, the fracture profile is enlarged by both the action of the viscosity dominated regime and plastic yielding.

This is result is supported by figure (6b) that presents the fluid pressures inside the fracture. Higher fluid pressures are observed, presenting highly non-uniform profile with the larger loading values being at the fracture inlet and smaller loading at the fracture tip. This type of fluid loading has a direct effect on the elliptic shape of the fracture. This behaviour is responsible for wider profiles.

When examining the net pressures of figure (6c), a small increase is created just after the injection starts suggesting pressure build up. This pressure is associated with the plastic zone development. After the plastic zones have developed, the pressure starts to decay. This behavior is similar for all cases examined with different values of fluid viscosity. Immediately after formation breakdown, the net pressures evaluated from the highly viscous cases tend to elevate. For small viscosities, the fluid diffuses freely in the formation and the pressure tends to a constant value. For high viscosities the diffusion process is affected and the pressure increases. This behaviour is explained by the fact that the fracture is driven in the viscosity dominated regime and furthermore from the plasticity considered in the models.

Figure (6d) shows the energy that is dissipated from plasticity versus time for all the cases examined. It is showed that more time is needed to propagate the fracture to a certain extend ( $1.5 \mathrm{~m}$ in length) when is driven with highly viscous fluids. The plastic dissipation curves follow the same path. From this plot we show that when the fracture is driven with highly viscous fluids, larger plastic zones are obtained as a result of the viscosity dominated fracture. For small fluid viscosity the plastic dissipation is almost the same and reaches the same fracture length at the same time.

We conclude that the obtained results are a direct result of the action of the viscous fluid flow which in turn affects the plastic zone development resulting in wider fracture profiles and larger fluid pressures compared to the poroelastic case. Furthermore, this phenomenon is amplified with the time scale difference between the diffusion process and the pumping schedule imposed with the highly viscous fluids used.

\section{CONCLUSIONS}

- A set of fully coupled poroelastic and poroplastic models have been presented.

- From the comparison with asymptotic solutions and other numerical studies we have demonstrated that our models are capable for predicting fluid driven fractures in toughness dominated and viscosity dominated regimes with excellent accuracy.

- When the fractures are driven with high flow rates under poroelastic conditions the fluid pressures turn non-uniform inside the fracture because the time scale of the fracture fluid diffusion is significantly different from the time scale of the fluid injection. As a result of 
the small diffusion that is allowed from the fracture towards the formation the obtained fracture profiles are wider and the net pressures after breakdown increase.

- When the fractures are driven with high flow rates under poroelastoplastic conditions the fluid pressures preserve their non-uniform profile inside the fracture for the same reason as the poroelastic case. As a result larger plastic yielding is created when the fracture is driven with high flow rates suppressing even more the diffusion process. The fracture profiles obtained under poroelastoplastic conditions are wider from the poroelastic case. Furthermore, the fluid pressures needed to extend the fracture are larger and the net pressures after break down increase as a result of the diffusion process.

- Hydraulic fracturing in the viscosity dominated regime under poroelastic conditions present similar behavior. When the fractures are driven with highly viscous fluids under poroelastic conditions the fluid pressures turn non-uniform inside the fracture. This is done because of the action of the viscous fluid flow. This type of flow affects the diffusion and the fluid is not allowed to diffuse properly in the formation thus increasing the pressure at the fracture inlet and decreasing the pressure near the tip area. This has a direct result over the elliptic shape of the fracture. As a result of the small diffusion that is allowed from the fracture towards the formation the obtained fracture profiles are wider and the net pressures after breakdown increase.

- Hydraulic fracturing in the viscosity dominated regime under poroelastoplastic conditions influence even further the results. When the fractures are driven with highly viscous fluids under poroelastoplastic conditions the fluid pressures turn non-uniform inside the fracture as a result of the action of the viscous fluid flow. This type of flow affects the diffusion and the fluid is not allowed to diffuse properly in the formation thus increasing the pressure at the fracture inlet and decreasing the pressure near the tip area. This nonuniform pressure creates larger plastic zones which in turn affects the elliptic shape of the fracture. The resulting fracture profiles are wider, the fluid and net pressures are larger from the poroelastic case. Furthermore, the net pressures increase after breakdown as a result of the action of the highly viscous fluid flow.

\section{ACKNOWLEDGEMENTS}

This work was co-funded by the European Regional Development Fund and the Republic of Cyprus through the Research Promotion Foundation (Project: DIDAKTOR/0311/58).

\section{REFERENCES}

[1] P. Valko, M.J. Economides, Hydraulic fracture mechanics. John Wiley and Sons, New York, 1995.

[2] V.C. Tsai, J.R. Rice, A model for turbulent hydraulic fracture and application to crack propagation at glacier beds. Journal of Geophysical Research, 115, 1-18, 2010.

[3] J. Adachi, E. Siebrits, A. Peirce, J. Desroches, Computer simulation of hydraulic fractures. International Journal of Rock Mechanics and Mining. Sciences, 44, 739-757, 2007.

[4] J. Hu, D.I. Garagash, Plane strain propagation of a fluid-driven crack in a permeable rock with fracture toughness. ASCE Journal of Engineering Mechanics, 136, 1152-1166, 2010.

[5] B. Lecampion, E. Detournay, An implicit algorithm for the propagation of hydraulic fracture with a fluid lag. Computer Methods in Applied Mechanics and Engineering, 196, 4863-4880, 2007. 
[6] J.Adachi, E. Detournay, Plain strain propagation of a hydraulic fracture in permeable rock. Engineering Fracture Mechanics, 75, 4666-4694, 2008.

[7] D.B.Van Dam, P. Papanastasiou, C.J. De Pater, Impact of rock plasticity on hydraulic fracture propagation and closure. Journal SPE Productions \& Facilities, 17, 149-159, 2002.

[8] E. Sarris, P. Papanastasiou, Modeling of hydraulic fracturing in a poroelastic cohesive formation. International Journal of Geomechanics, 12, 160-167, 2012a.

[9] A.P. Bunger, E. Detournay, D.I. Garagash, Toughness-dominated hydraulic fracture with leak-off. International Journal of Fracture, 134, 175-190, 2005.

[10] J.T. Boone, A.R. Ingraffea, A numerical procedure for simulation of hydraulically driven fracture propagation in poroelastic media. International Journal for Numerical and Analytical Methods in Geomechanics, 14, 27-47, 1990.

[11] P. Papanastasiou, The influence of plasticity in hydraulic fracturing. International Journal of Fracture, 84, 61-79, 1997.

[12] P. Papanastasiou, An efficient algorithm for propagating fluid driven fractures. Computational Mechanics, 24, 258-267, 1999a.

[13] J.M. Segura, I. Carol, On zero-thickness interface elements for diffusion problems. International Journal for Numerical and Analytical Methods in Geomechanics, 28, 947-962, 2004.

[14] E. Sarris, P. Papanastasiou, The influence of cohesive process zone in hydraulic fracturing modelling. International Journal of Fracture, 167, 33-46, 2011.

[15] E.Sarris, P. Papanastasiou, Numerical modeling of fluid-driven fractures in cohesive poroelastoplastic continuum. International Journal for Numerical and Analytical Methods in Geomechanics, DOI: 10.1002nag.2111, 2012b.

[16] Z. Chen, Finite element modeling of viscosity-dominated hydraulic fractures. Journal of Petroleum Science and Engineering, Doi: 10.1016/j.petrol.2011.12.021, 2012.

[17] B. Carrier, G. Sylvie, Numerical modeling of hydraulic fracture problem in permeable medium using cohesive zone model. Engineering Fracture Mechanics, 79, 312-328, 2012.

[18] J.R. Rice, A path independent integral and the approximate analysis of strain concentration by notches and cracks. Journal of Applied Mechanics, 35, 379-386, 1968.

[19] Abaqus, Version 6.10 Theory Manual, Providence, RI, 2010.

[20] A. Peirce, E.Detournay, An implicit level set method for modeling hydraulically driven fractures. Computer Methods in Applied Mechanics and Engineering, 197, 2858-2885, 2008.

[21] P. Papanastasiou, M. Thiercelin, Influence of inelastic rock behavior in hydraulic fracturing. International Journal of Rock Mechanics, Mining Sciences and Geomechanical Abstracts, 30, 1241-1247, 1993.

[22] V. Tomar, J. Zhai, M. Zhou, Bounds for element size in a variable stiffness cohesive finite element model. International Journal for Numerical Methods in Engineering, 61, 18941920, 2004. 